\title{
LESIÓN ÓRBITO-CRANEAL PENETRANTE TRAS TRAUMATISMO PALPEBRAL MÍNIMO: A PROPÓSITO DE UN CASO
}

\section{PENETRATING ORBITOCRANIAL INJURY AFTER TRIVIAL EYELID TRAUMA: A CASE REPORT}

\author{
RUIZ-MARTÍN MMํㄹ , FERNÁNDEZ-PRIETO A ${ }^{2}$, VIÉITEZ-VÁZQUEZ J ${ }^{1}$, \\ MOSQUERA-LEZCANO $\mathrm{R}^{1 \dagger}$
}

\section{RESUMEN}

Caso clínico: Presentamos un varón en estado de embriaguez con una hemorragia cerebral asociada a una herida palpebral mínima tras agresión con objeto metálico.

Discusión: Una aparente lesión palpebral banal puede acompañarse de lesiones cerebrales graves. Es fundamental hacer un correcto diagnóstico diferencial entre embriaguez y daño orgánico, dadas las repercusiones que puede conllevar, tanto a nivel clínico como médico-legal.

Palabras clave: Hemorragia cerebral, herida palpebral penetrante, objeto metálico, embriaguez, agresión.

\begin{abstract}
Case report: We report a patient who, while in a drunken state, sustained a cerebral hemorrhage associated with an apparently trivial eyelid injury after an aggressive attack with a metallic object.

Discussion: Any apparently trivial eyelid injury may be associated with serious cerebral lesions. Making a correct diagnosis that distinguishes between that of drunkenness and an organic injury is essential, because failure to do so may result in both clinical and legal repercussions (Arch Soc Esp Oftalmol 2007; 82: 785-788).
\end{abstract}

Key words: Cerebral hemorrhage, penetrating eyelid injury, metallic object, drunkenness, aggression.

\section{INTRODUCCIÓN}

Las lesiones órbito-frontales son frecuentemente pasadas por alto debido a su escasa sintomatología clínica (1). Si a esto añadimos el fétor etílico como factor de confusión, podemos obviar su diagnósti- co, con las graves consecuencias tanto clínicas como médico-legales que de ello pueden derivar.

En la actualidad, junto a una buena exploración neurológica, la contribución de la Tomografía Computerizada (TC) en las urgencias hospitalarias supone un arma diagnóstica útil e indispensable, pues

\footnotetext{
Recibido: 16/5/06. Aceptado: 17/7/07.

Servicio de Oftalmología. Hospital La Paz. Madrid. España.

1 Licenciado en Medicina.

2 Licenciado en Medicina. Servicio de Radiología.

Comunicación presentada en el LXXVII Congreso de la S.E.O. (Barcelona 2001).

Correspondencia:

M. ${ }^{a}$ del Mar Ruiz Martín

C/. Granada, 3

18184 Beas de Granada (Granada)

España

E-mail: mmruizmm@hotmail.com
} 
además de demostrarnos la extensión del daño órbito-craneal, nos indica el mecanismo de la lesión, su trayectoria y la presencia y localización de fragmentos óseos y/o metálicos. También sirve para la monitorización del daño $(2,3)$.

\section{CASO CLÍNICO}

Presentamos un varón de 35 años que acude a urgencias por agresión en el párpado superior izquierdo con un objeto metálico, fino y largo, mientras deambulaba por la calle. Presenta importante fétor etílico e inestabilidad en la marcha.

En la exploración oftalmológica encontramos una agudeza visual (AV) en el ojo izquierdo (OI) poco fiable por la pésima colaboración del paciente, pero que al menos es de 0,7. La motilidad ocular intrínseca y extrínseca son normales. No refiere diplopía en ninguna posición de la mirada.

Se objetiva un hematoma palpebral superior con una pequeña herida inciso-contusa, superficial, que no precisa punto de sutura.

La biomicroscopía, tensión ocular y fondo de ojo son normales.

Se deja al paciente en observación, presentando en las horas siguientes un deterioro neurológico progresivo, consistente en desorientación temporoespacial, respuestas incoherentes, somnolencia progresiva y vómitos de repetición, por lo que se le realiza la Escala del Coma de Glasgow (GCS), que resulta de 12, una TC orbitaria (fig. 1), donde se aprecia un aumento de partes blandas periorbitario izquierdo correspondiente a un hematoma y un fragmento óseo con fractura asociado en el margen superoposterior y lateral de la órbita izquierda, y una TC cerebral (fig. 2), que muestra un hematoma agudo intraparenquimatoso frontal izquierdo que se abre al asta anterior del ventrículo lateral adyacente y al resto del sistema ventricular y una hemorragia subaracnoidea en la cisura de Silvio izquierda, surcos de la convexidad izquierda, cisterna supraselar y perimesencefálicas.

Se practica una analítica, que descarta cualquier tendencia hemorrágica del paciente (tiempo de sangrado, tiempo de protrombina, tiempo de tromboplastina parcial activada, fibrinógeno y plaquetas normales).

Ante la posibilidad de un vaso sangrante activo así como de un posible daño arterial, se realiza una arteriografía por sustracción digital (ASD) cerebral, en la cual no se encuentran hallazgos patológicos (fig. 3).

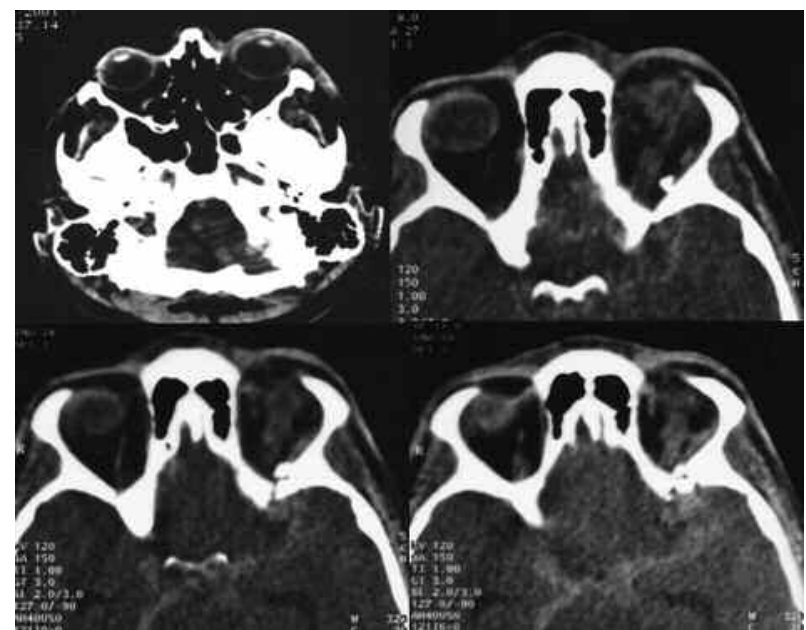

Fig. 1: TAC orbitario (corte axial): se aprecia un aumento de partes blandas (hematoma) periorbitario izquierdo y un fragmento óseo con fractura asociado en el margen superoposterior y lateral de la órbita izquierda.

El paciente es ingresado en la Unidad de Cuidados Intensivos. Durante el ingreso se realizan TC y ASD de control en los días 1, 2, 3, 7 y 14, que descartan nuevos resangrados ó lesiones vasculares. El paciente evoluciona favorablemente y es dado de alta con una GCS de 15. La TC al alta muestra un hematoma frontal izquierdo en resolución con pequeño componente intraventricular lateral frontal

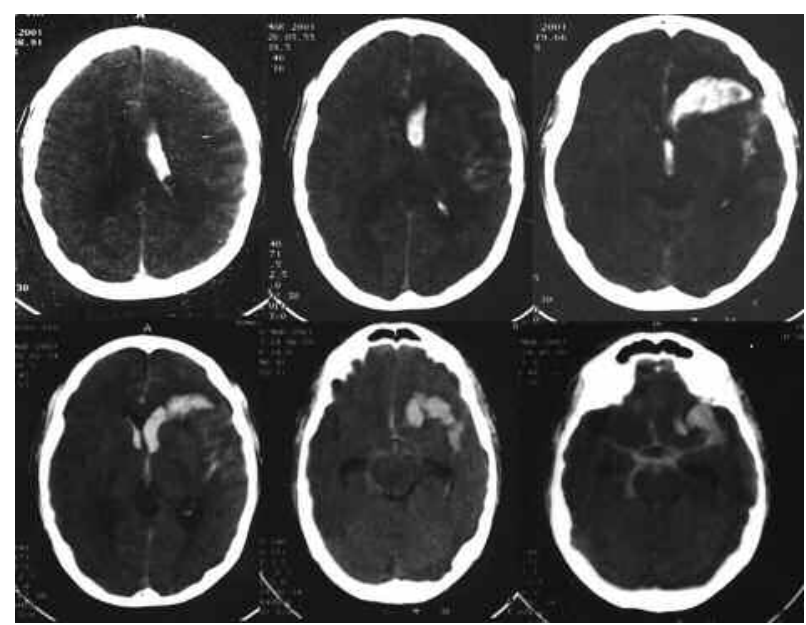

Fig. 2: Hematoma agudo intraparenquimatoso frontal izquierdo, que se abre al asta anterior del ventrículo lateral adyacente y al resto del sistema ventricular y una hemorragia subaracnoidea en la cisura de Silvio izquierda, surcos de la convexidad izquierda, cisterna supraselar y perimesencefálicas. 


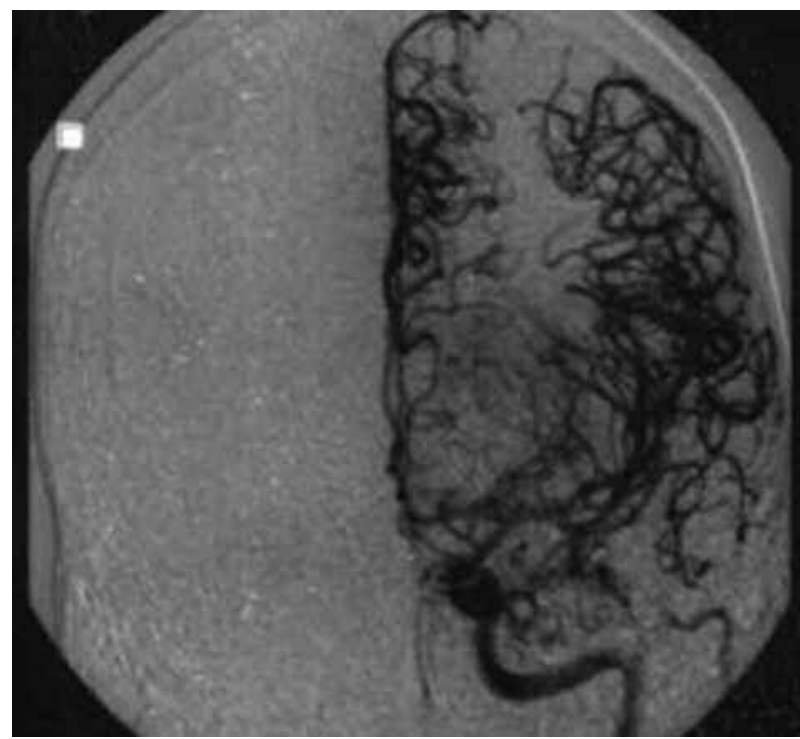

Fig. 3: Arteriografía por Sustracción Digital (ASD) cerebral con cateterismo selectivo de arterias carótidas internas: sin hallazgos patológicos.

izquierdo y restos hemáticos isodensos en la cisura de Silvio izquierda (fig. 4).

\section{DISCUSIÓN}

Las lesiones cerebrales penetrantes por cuerpo extraño más frecuentemente descritas en la literatura son por arma de fuego, y, con mucha menor frecuencia, por otros tipos de objetos. También se han descrito heridas periorbitarias por cuerpo extraño, pero que el objeto causante de la lesión cerebral entre atravesando la órbita es un hallazgo extraordinariamente raro (1-3). Además existen diferentes tipos de material, con la distinta susceptibilidad de cada uno a complicaciones como la infección ó la reacción tisular. En general, el cerebro tolera bastante bien los objetos metálicos, aunque existen casos descritos de abscesos cerebrales incluso años después del traumatismo (1).

La órbita tiene una especial configuración cuyas paredes convergentes tienden a guiar los objetos penetrantes hacia el ápex. Como la cabeza se extiende instintivamente hacia atrás en el momento del traumatismo en una actitud defensiva, es bastante frecuente que fracture el techo de la órbita y penetre en el lóbulo frontal $(1,2)$. Pese a su gravedad, estas lesiones pasan frecuentemente desaperci-

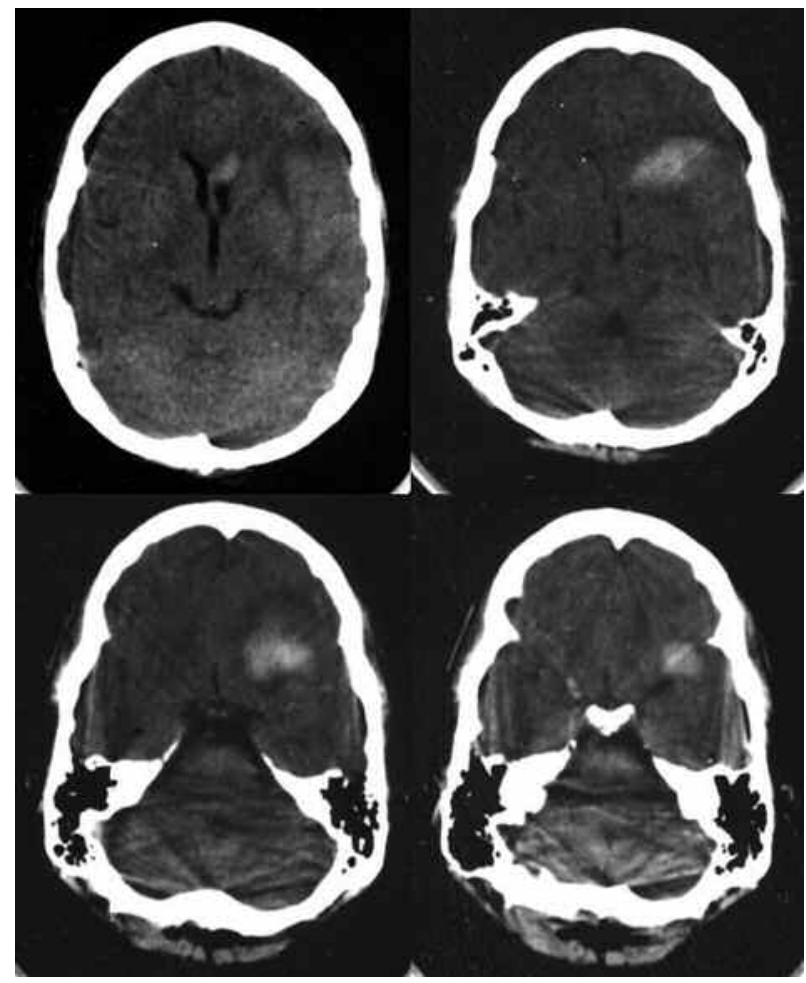

Fig. 4: Hematoma frontal izquierdo en evolución ( sangre de menor valor de atenuación que los TC previos), con pequeño componente intraventricular lateral frontal izquierdo. Restos hemáticos isodensos en cisura de Silvio izquierda.

bidas por el marcado hematoma periorbitario y los leves signos neurológicos (1). Se añade, en nuestro caso, el estado de embriaguez que nos falsea los posibles signos de lesión cerebral.

Además, aunque la anisocoria es lo habitual, existen excepciones a la regla, por lo que una exploración de los reflejos pupilares fotomotores normal no descarta siempre una posible afectación cerebral.

Por tanto, atender a las características del objeto agresor en cuanto a material, forma, tamaño y trayectoria nos puede ayudar a dilucidar el mecanismo lesional y su posible gravedad.

Besenski $\mathrm{N}$ et al. reportan que el tipo de lesión cerebral penetrante más frecuente apreciado por TC es la hemorragia (84\%) seguido de hallazgos de fractura con fragmentos óseos (67\%) (3). Fujitsu et al. revelan que la causa más frecuente de hemorragia intraventricular traumática es un hematoma intracerebral en el lóbulo frontal ó temporal extendiéndose hacia el cuerno anterior ó inferior del ventrículo lateral, ocurriendo con un retraso de 6 a 12 horas (4). 
Un traumatismo cerebral puede complicarse, en raras ocasiones, con lesiones arteriales intracraneales traumáticas, que incluye las disecciones arteriales, los pseudoaneurismas y las fístulas arteriovenosas. Se debe tener siempre en mente la posibilidad de dichas lesiones vasculares para prevenir cualquier complicación hemorrágica que puede ser potencialmente letal. De ahí la importancia de la ASD (5), tanto en el momento inicial como en el seguimiento del paciente.

De todas formas, la prueba diagnóstica estrella para el oftalmólogo de urgencias que se encuentra ante un traumatismo palpebral penetrante y de profundidad desconocida es la TC, pues la gran capacidad de la región orbitaria para el edema y la hemorragia y los escasos signos neurológicos pueden enmascarar una afectación cerebral concomitante, en especial ante signos de embriaguez.

En conclusión, la TC es un arma indispensable e irreemplazable, incluso cuando la lesión es aparentemente superficial, tanto en la valoración inicial para mostrarnos la presencia, extensión y mecanismo del daño órbito-craneal, como en el seguimiento del paciente $(1,3)$.

\section{BIBLIOGRAFÍA}

1. Scarfo GB, Mariottini A, Palma L. Oculocerebral perforating trauma by foreign objects: diagnosis and surgery. $J$ Neurosurg Sci 1990; 34: 111-116.

2. Ildan F, Bagdatoglu H, Boyar B, Doganay M, Cetinalp E, Karadayi $A$. The nonsurgical management of a penetrating orbitocranial injury reaching the brain stem: case report. J Trauma 1994; 37: 116-118.

3. Besenski N, Jadro-Santel D, Jelavic-Koic F, Pavic D, Mikulic D, Glavina K, et al. CT analysis of missile head injury. Neuroradiology 1995; 37: 207-211.

4. Fujitsu K, Kuwabara T, Muramoto M, Hirata K, Mochimatsu $Y$. Traumatic intraventricular hemorrhage: report of twenty-six cases and consideration of the pathogenic mechanism. Neurosurgery 1988; 23: 423-430.

5. Tsutsumi M, Kazekawa K, Tanaka A, Ueno Y, Nomoto $Y$, Nii $K$, et al. Traumatic middle meningeal artery pseudoaneurysm and subsequent fistula formation with the cavernous sinus: case report. Surg Neurol 2002; 58: 325-328. 